\title{
Langue et vulnérabilité des migrations chinoises actuelles
}

\section{Estelle Auguin et Florence Levy}

\section{(2) OpenEdition}

1 Journals

Édition électronique

URL : https://journals.openedition.org/remi/4205

DOI : $10.4000 /$ remi.4205

ISSN : $1777-5418$

Éditeur

Université de Poitiers

\section{Édition imprimée}

Date de publication : 1 décembre 2007

Pagination : 67-84

ISBN : 978-2-911627-47-4

ISSN : 0765-0752

\section{Référence électronique}

Estelle Auguin et Florence Levy, "Langue et vulnérabilité des migrations chinoises actuelles », Revue européenne des migrations internationales [En ligne], vol. 23 - n³ | 2007, mis en ligne le 01 décembre 2010, consulté le 15 avril 2022. URL : http://journals.openedition.org/remi/4205 ; DOI : https://doi.org/ 10.4000/remi.4205

Ce document a été généré automatiquement le 15 avril 2022.

(c) Université de Poitiers 


\title{
Langue et vulnérabilité des migrations chinoises actuelles
}

\author{
Estelle Auguin et Florence Levy
}

1 Jusqu'à une période récente, l'immigration chinoise ne posait pas de problème à la société française qui l'observait comme discrète et économiquement bien intégrée. On la considère parfois comme une communauté ${ }^{1}$ étrangère "idéale » car perçue comme calme et autorégulée, ne nécessitant que rarement l'intervention des autorités. Cependant, depuis la fin des années quatre-vingt-dix, les choses ont changé. En particulier l'apparition des Chinois sur la scène publique lors des manifestations de sans-papiers en 1997, surtout dans le Troisième collectif des sans-papiers, a surpris et rendu visible une immigration illégale dont l'ampleur était jusqu'alors insoupçonnée. La société voit apparaitre des signes de difficultés : les pouvoirs publics constatent, par exemple, une nette augmentation de la population chinoise dans les services sociaux (Caisse d'Allocations Familiales, hôpitaux, etc.) et la multiplication des interventions de la police suscite des préoccupations. Ce phénomène s'expliquerait principalement par une diminution des capacités d'intégration communautaire due à une importante augmentation du nombre de migrants et à la diversification de leurs origines. Les ressources internes à la communauté ne semblent plus suffisantes pour résoudre l'ensemble des problèmes de ses membres et de plus en plus de personnes se tournent vers l'extérieur, c'est-à-dire vers la société française.

2 Quels facteurs permettent d'expliquer l'augmentation de la vulnérabilité de la population chinoise ? L'hypothèse principale de cet article est de considérer, qu'étant donné l'importance attribuée à la maîtrise de la langue française dans le modèle d'intégration français, le facteur linguistique devient alors central pour comprendre la vulnérabilité des primo-arrivants chinois. En effet, la maîtrise de la langue française conditionne la sociabilité des migrants dans la société d'accueil et leur accès direct à cette dernière. Or, les migrants adultes d'origine chinoise rencontrent de grandes difficultés dans leur apprentissage et finissent souvent par y renoncer. En l'absence de compréhension linguistique, ils font appel à des médiateurs appartenant à la communauté chinoise qui servent d'intermédiaire entre les individus et la société 
française. Aussi, le sas communautaire devient un espace vital pour ces migrants qui vont y puiser tous les éléments pour assurer leur survie et leur installation dans le nouveau pays.

Notre propos est basé sur des recherches empiriques ${ }^{2}$ qui portent sur deux ${ }^{3}$ populations chinoises vivant en France, en particulier dans la région parisienne ${ }^{4}$. La première, en provenance de la région méridionale de Wenzhou, est installée en France depuis plus d'un demi-siècle et possède un ancrage communautaire fort. Ce flux, composé de jeunes hommes et femmes ruraux, faiblement qualifiés et lourdement endettés, s'inscrit dans une chaîne migratoire familiale qui s'est amplifiée essentiellement depuis le milieu des années quatre-vingt. La seconde population arrivée depuis la fin des années quatrevingt-dix des villes du Nord de la Chine, et du Dongbei (la région du Nord-Est), ne possède pas en France de réseau communautaire d'entraide. Ce groupe est essentiellement composé de citadins quadragénaires - majoritairement des femmes, souvent divorcées - ayant appartenu à une classe moyenne maintenant déclassée, et se démarque nettement du premier courant numériquement majoritaire. Ces deux populations, très différentes par les profils et les projets migratoires, ont pour point commun de se retrouver en France, au moins dans un premier temps, sans-papiers ${ }^{5}$ et avec une faible maîtrise des rudiments du français.

\section{De l'importance de la langue dans le modèle d'intégration républicain...}

4 À la fin du $\mathrm{XX}^{\mathrm{e}}$ siècle, pratiquement tous les pays européens sont pourvus d'une langue nationale. Depuis, plus d'un siècle, préside l'équation une langue = une nation (Thiesse, 1999 : 67). Cette idée est apparue indispensable voire constitutive du projet politique de la France dès la Révolution. Aujourd'hui, cela a des implications importantes pour l'accueil des migrants.

\section{La langue française et la nation}

\section{La langue française, un facteur d'unité politique}

5 L'une des fonctions principales d'une langue nationale est "d'incarner la nation », «quelle que soit leur origine géographique et sociale, tous ces membres doivent la comprendre et l'utiliser" (Thiesse, 1999: 70-71). Si cela est vrai dans la majorité des nations modernes, cette importance linguistique a en France une portée politique. Avec la Révolution française, la diversité linguistique du territoire est perçue comme une menace pour l'unité politique, c'est-à-dire idéologique (Weber, 1983 : 114). «L'idéal de la Révolution visait à l'uniformité et à la suppression des particularismes » dans le but d'unifier un peuple autour d'un projet politique, d'où l'importance que chacun des enfants connaisse le langage de la République, «le langage de le Déclaration des Droits » (Weber, 1983 : 115). Et si cette politique fut un échec dans le courant du XIX ${ }^{\mathrm{e}}$ - il faut attendre le début du XXe siècle pour que la majorité de la population connaisse le français -, le principe de l'unité linguistique est posée : «l'usage de la langue de la nation [devient alors] un devoir » (Thiesse, 1999 : 70).

6 Or, «la nation française est fortement marquée par la dimension politique » et la Révolution comme «mythe fondateur» (Schnapper, 1991: 41). Dans cette idée de la 
nation «qu'on peut appeler "contractuelle" ", « les responsables ont pour ambition de renforcer l'homogénéité culturelle qui contribue à fonder et à entretenir la dimension communautaire de la nation » (Schnapper, 1991 : 107). Aussi, le français comme langue commune est-il aujourd'hui un fait acquis. Le consensus national est sur ce point pratiquement total; il suffit pour cela de lire la première phrase de l'article 2 de la Constitution du 4 octobre 1958 de la Ve République qui précise que «La langue de la République est le français. ».

\section{La langue française, un facteur d'intégration}

7 Contrairement à ses voisins européens, la France n'a jamais été une terre d'émigration, mais, au contraire, a connu un déclin démographique dès le XIX ${ }^{e}$ siècle, faisant d'elle un territoire d'immigration. Toutefois, « la méconnaissance systématique de l'immigration et des mécanismes d'intégration a été [...] l'un des moyens permettant d'entretenir l'unité nationale.» (Schnapper, 1991 : 13). Étant donné le projet politique de la France, qui fonde son idée de la nation sur la volonté des citoyens, le terme d'ethnie a toujours été refusé pour prôner a contrario une homogénéité culturelle. Si aujourd'hui la France comme pays d'immigration est une idée acceptée, les différentes origines des migrants sont rarement prises en compte afin de privilégier le maintien de l'homogénéité comme objectif. Cela conduit à traiter tous les migrants qui souhaitent s'installer sur son sol comme des individus qui adhèrent au projet républicain sans se préoccuper de leur origine ethnique. Cette homogénéité passe par l'usage d'une langue commune.

8 Longtemps cette injonction d'intégration ne passait pas par une action concrète des pouvoirs publics qui s'en remettait aux démarches individuelles des migrants. En 2003, le contrat d'accueil et d'intégration (CAI) est mis en œuvre dans quelques départements pilotes, puis généralisé à l'ensemble du territoire en 2005. Il est depuis systématiquement proposé à tout nouvel arrivant régulier ou lors de la régularisation de personnes clandestines. L'accent est mis sur les droits et les obligations réciproques des deux parties, que ce soit de l'État français ou du nouvel arrivant. Dans son préambule, il est indiqué que "choisir de vivre en France, c'est avoir la volonté de s'intégrer à la société française. » Cette injonction est complétée de cinq éléments mis en exergue pour présenter la France et définir son projet politique comme étant 1) une démocratie, 2) un pays de droits, 3) un pays laïque, 4) un pays d'égalité. Enfin, le cinquième point affirme que «la connaissance du français est le premier atout de l'intégration. " Si le migrant accepte de signer ce contrat, il s'engage notamment à apprendre le français. Des cours de langue lui sont gracieusement offerts ; l'assiduité à ces cours est contrôlée et à défaut considérée comme un manquement aux obligations du contrat ${ }^{6}$.

9 Les administrations françaises ne distinguent pas les migrants selon leurs origines et semblent considérer que s'adapter à leurs spécificités relèverait d'un traitement de faveur qui serait incompatible avec le principe d'égalité. Cela conduit les administrations françaises à traiter les migrants chinois de la même manière que ceux d'origine européenne ou provenant de régions francophones et/ou anciennement colonisées. Ces derniers sont, au-delà de la connaissance de la langue, plus familiarisés avec le système institutionnel français. La majorité des Chinois paraissent, quant à eux, mal armés dans cet échange et leur faible maîtrise de la langue française et des modes de fonctionnement du système sont à l'origine de nombreux malentendus avec les pouvoirs publics. 


\section{La difficulté linguistique des Chinois face au modèle d'intégration}

\section{Aucun repère linguistique}

10 dans leur langue et, dans le cas des Wenzhou, sont habitués à jongler entre leur dialecte et le mandarin ${ }^{7}$, éprouvent de grandes difficultés à apprendre le français. Deux raisons peuvent expliquer ce fait.

11 Les deux langues appartiennent à des systèmes linguistiques fondamentalement différents par leur phonétique, leur syntaxe et leur écriture, ce qui rend l'apprentissage du français extrêmement difficile. Les migrants chinois habitués à distinguer les phonèmes vocaliques déclinées selon quatre tons, peinent $a$ contrario à distinguer les sons du français et à les prononcer. La logique de la construction des deux langues est profondément différente. Habitués à une construction qui fait sens avec des caractères, des idéo-phonogrammes ${ }^{8}$, les migrants ont du mal à comprendre la logique " abstraite " et phonétique de l'alphabet qui leur est profondément étrangère. D'autre part, la structure grammaticale du chinois est relativement simple; les caractères, toujours invariables, s'agencent de manière flexible et ne se déclinent jamais. Par conséquent, la rationalité compliquée du système grammatical français qui fonctionne sur des déclinaisons sans cesse changeantes leur est difficilement assimilable : les conjugaisons, inexistantes en chinois, sont une étape souvent difficile dans l'apprentissage ; la notion de temps ne s'exprime pas de la même manière en chinois et ne recouvre pas les catégories des temps du français. Ainsi en France, les malentendus sont extrêmement nombreux du fait d'un emploi erroné de la conjugaison ou de l'expression du temps.

Ces primo-arrivants chinois n'a jamais appris le français avant le départ. Contrairement à d'autres migrants originaires de pays appartenant à la sphère francophone, ils n'ont jamais eu l'occasion en Chine de se familiariser avec cette langue, ni avec aucune autre langue européenne. Si de nos jours, l'anglais est enseigné à l'école primaire et au collège, le niveau de l'enseignement est souvent extrêmement faible et la plupart des Chinois présents en France ont été scolarisés avant cette réforme. Cette méconnaissance totale des langues européennes est un handicap particulier. Si aujourd'hui l'alphabet latin est enseigné aux enfants chinois à travers notamment le pinyin, c'est-à-dire la transcription phonétique en lettres latines des caractères chinois, nombreux sont les migrants chinois qui maîtrisent mal l'alphabet, voire qui ne le connaissent pas du tout, puisqu'aucun usage n'en est fait en dehors du milieu scolaire ou de l'usage de la micro-informatique. Dès lors, une fois en France, déchiffrer toute information en Français devient une épreuve. Les actions quotidiennes comme celles de se repérer dans une rue ou sur un plan de métro sont délicates et s'aventurer à l'extérieur de son quartier représente un souci supplémentaire que certains migrants préfèrent éviter 9 .

13 Les difficultés d'apprentissage de la langue française sont donc importantes. Après quelques années en France, ceux qui ont eu l'occasion de côtoyer des Français ou d'autres étrangers ont réussi à acquérir des rudiments de la langue française. Il s'agit le plus souvent de mots répétés avec une prononciation approximative et dont le sens est incertain mais suffisant pour la plupart des interactions quotidiennes. Les femmes de ménage apprennent par exemple le vocabulaire lié à la maison et au nettoyage, tandis que les commerçants finissent par savoir compter et négocier en français. Mais leur 
niveau reste trop limité pour garantir une compréhension fiable notamment lors de contacts avec les administrations françaises.

\section{Un apprentissage long et parfois impossible}

14 Cependant, la majorité des migrants rencontrés souhaitent apprendre le français. La demande semble très forte comme en témoigne l'accroissement du nombre d'écoles de langue payantes qui ont ouvert leurs portes depuis la fin des années 1990. L'offre est pléthorique et en grande partie communautaire: les écoles sont ouvertes par des Chinois, pour des Chinois, avec des enseignants chinois. Il semble que les migrants se sentent rassurés par la présence d'enseignants sinophones capables de leur donner des explications dans leur langue maternelle. Les cours s'adressent à la fois aux adultes et aux enfants ${ }^{10}$. L'inscription est payante mais abordable, en moyenne légèrement supérieure à un euro de l'heure.

15 Les stratégies d'apprentissage des migrants se différencient selon les sexes, l'âge, l'origine géographique ainsi que les projets migratoires. Certaines personnes s'inscrivent dès les premiers jours de leur arrivée en France, en particulier les migrants du Nord. "Si tu es en France, alors tu dois apprendre le français.» D'autres, comme beaucoup de Wenzhou, se consacrent d'abord au remboursement de leur dette, puis une fois allégés de ce poids, se dirigent vers les écoles. On remarque que les jeunes adultes wenzhou sont très nombreux. Quant aux migrants du Nord, les écoles sont plus fréquentées par les femmes dont le projet migratoire a évolué et qui souhaitent dès lors s'établir en France par le biais du mariage, que par des hommes majoritairement non divorcés au pays, qui souhaitent à moyen terme retourner vivre en Chine et investissent moins dans l'apprentissage de la langue.

Trois facteurs sociaux permettent d'expliquer les difficultés d'apprentissage. D'une part, le niveau d'éducation scolaire est relativement bas chez les Wenzhou, qui ont pour le plus grand nombre arrêtés les études après l'école primaire ou le collège. Il y aurait $20 \%$ d'analphabètes dans cette population (Pina-Guerassimoff, 2002). Leur capacité à fournir un travail intellectuel exigeant un effort d'abstraction est de ce fait peu entraînée. Quant aux personnes originaires du Nord de la Chine, elles évoquent le facteur de l'âge pour expliquer les difficultés rencontrées; à plus de 40 ans, elles ont du mal à assimiler un cadre linguistique et de pensée si différent de celui qu'elles ont connu jusque-là. Enfin, pour les deux groupes, les conditions de vie et les projets migratoires influent fortement sur le processus d'apprentissage. Les migrants ont du mal à arbitrer entre des contraintes de temps contradictoires : consacrer leur temps à travailler pour rembourser leur dette et économiser de l'argent, ou au contraire investir dans un processus long d'apprentissage linguistique. Beaucoup suivent leur formation après une journée de labeur et arrivent à l'école exténués. S'ils parviennent à assister régulièrement aux cours, ils ont en revanche du mal à trouver le temps pour réviser et intégrer au fur et à mesure les connaissances. De nombreuses personnes apprennent le français par intermittence, profitant d'une période d'inactivité pour s'inscrire et interrompant leurs sessions dès qu'elles retrouvent un emploi. De plus, les conditions de logement, en dortoirs collectifs et surpeuplés, n'offrent pas non plus un cadre d'apprentissage optimum.

17 Beaucoup de migrants rencontrés, convaincus d'une difficulté excessive du français répètent: "le français, c'est trop difficile; je n'arrive pas à l'apprendre». Nous avons pu constater dans les différentes écoles où nous avons donné des cours, que la proportion 
de personnes ayant acquis les bases du français ou même un niveau suffisant pour communiquer sans obstacle, reste peu importante. Nombreux sont les abandons sans avoir assimilé les connaissances élémentaires. Ces personnes vont par la suite se contenter d'une communication minimale avec la population française et souvent mettre en place plus ou moins inconsciemment des stratégies d'évitement.

Un autre facteur semble avoir une influence décisive: la présence de nombreux compatriotes. On peut se demander si la communauté chinoise, en offrant par sa taille une alternative viable de sociabilité, ne permet pas aux migrants de contourner la difficulté linguistique. Face à un modèle d'intégration à la nation française qui se focalise sur la connaissance de la langue française, les migrants qui ont des difficultés inhérentes à leurs parcours socio-culturels et linguistiques, sont fragilisés dans la société d'accueil et cherchent des solutions pour pallier cette vulnérabilité.

\section{... à la vulnérabilité de la population chinoise}

\section{Le repli communautaire : une réponse aux difficultés linguistiques}

\section{Le sas communautaire}

19 Le problème de la langue peut être en effet contourné par l'installation dans un espace communautaire. Les migrants chinois - comme d'autres - ont su tirer parti d'un certain désintérêt de la société d'accueil et de l'écart linguistique entre le français et leur langue d'origine pour organiser librement un espace de vie. Ils ont mis en place, en exploitant cette zone grise délimitée par la langue, une vie intra-communautaire quasi exclusivement chinoise et relativement indépendante de la société française voire parfois de ses lois. Les nombreuses petites annonces d'offres d'emploi ou de services - tels que la traduction, la vente de cigarette, la location de lits en dortoirs ou les publicités pour des passeurs - en caractères chinois affichées dans les rues parisiennes des quartiers à forte population chinoise, illustrent bien ce phénomène.

Cet espace de vie offre une solution à l'urgence dans laquelle se trouvent les nouveaux migrants, urgence qui est peu compatible avec la temporalité longue de l'administration française ou même le temps de l'apprentissage de la langue. Dès leur arrivée, ils sont soumis à d'importantes pressions financières qui vont dicter leurs priorités. Le poids du remboursement de la dette contractée pour le voyage illégal (entre 18000 et 27000 euros) pèse sur les migrants en provenance du Sud de la Chine. Il leur faut entre 3 et 10 ans de travail journalier pour y parvenir. Pour les personnes du Nord, le coût du voyage est moins élevé et a parfois été déjà payé avec leurs propres économies. Leur projet ${ }^{11}$ n'est pas, au démarrage, de s'installer en France mais de gagner de l'argent pour revenir en Chine au bout de deux ou trois ans. Tous sont donc pressés à leur arrivée de trouver un logement pour pouvoir se consacrer à l'objectif de la migration : travailler et économiser au plus vite. Ce but est rarement perdu de vue, même si ses modalités vont progressivement se modifier. Les migrants savent que pour le réaliser, ils peuvent s'installer dans un "à côté » de la société française et se passer de l'apprentissage du français, du moins à court terme.

De fait, alors que l'accès à la société française est difficile, la communauté chinoise offre aux nouveaux arrivants des solutions à la majeure partie de leurs besoins urgents. C'est auprès de leurs compatriotes qu'ils vont pouvoir se procurer très rapidement un 
logement, souvent en dortoir. Ce lieu en fournissant un premier réseau de sociabilité jouera un rôle central dans l'obtention d'informations pratiques pour vivre en France et en particulier dans la recherche d'un emploi. Le travail se trouve le plus souvent par réseau, sur présentation (parfois payante) ou par l'intermédiaire d'annonces collées dans les rues ou publiées dans les journaux. Le marché de l'emploi est quasi exclusivement communautaire : à destination d'employés chinois et pour le compte de patrons d'origine chinoise. Les sphères professionnelles et commerciales sont d'ailleurs l'occasion d'un rare croisement entre Chinois d'outre mer, Wenzhou et personnes du Nord. Ce n'est que dans un deuxième temps que les migrants se dirigent vers des patrons appartenant à d'autres sphères communautaires comme les personnes d'origine turques ou de la communauté juive de Paris. À noter que la sortie de l'emploi ethnique marque une certaine mobilité professionnelle et salariale dont les migrants tirent fierté.

22 L'absence de permis de séjour se cumule au handicap linguistique et constitue une double barrière qui limite l'accès direct des migrants à la société française. En effet, l'insécurité liée à l'absence de statut légal imprègne leur vie quotidienne. Comme pour d'autres migrants, la précarité de la clandestinité conduit à la méfiance et au repli sur les semblables. Les conseils de ne pas sortir (pour éviter les contrôles policiers) et de fréquenter le moins possible des Français (dont on ne connaît pas les intentions) ne sont pas rares. Ne parlant pas le français, les contacts sont limités aux locuteurs sinophones. Enfin, le manque de capitaux culturels tels que la connaissance des modalités de socialisation en France ou tout simplement de réseaux sociaux contribue également à limiter les opportunités d'échanges et à circonscrire la vie quotidienne dans des limites « ethniques».

\section{La non-reconnaissance des communautés}

Ainsi si la présence d'une communauté sinophone est un atout permettant de contourner en partie les difficultés liées à la langue, a contrario vis-à-vis de la société française cela s'avère plutôt être un handicap. Les migrants sont confrontés à un nouveau problème: la non-reconnaissance des communautés par la République française.

En ce sens, la conception française de la nation inventée lors de la Révolution est une "conception rousseauiste d'une démocratie unitaire, hostile au pluralisme" (Schnapper, $2000: 44$ ). À l'opposé de la conception anglaise de la citoyenneté où l'on " est citoyen par l'appartenance à une communauté particulière. " (Schnapper, 2000 : 42), le projet politique français est l'homogénéité d'un peuple uni autour d'idéaux républicains et de valeurs universelles. Ces traditions continuent d'engendrer des pratiques différentes dans les politiques d'intégration des populations immigrées qui sont menées en Grande-Bretagne et en France. "Dans le premier cas, on accepte qu'existent des formes de communautés socialement - mais non juridiquement reconnues, dont les intérêts sont spécifiques et dont les représentants négocient avec les pouvoirs publics pour obtenir des droits particuliers. Dans le second, on continue à s'efforcer d'intégrer les populations d'origine étrangère par la citoyenneté individuelle, en refusant de reconnaître l'existence de communautés particulières dans l'espace public. » (Schnapper, $2000: 50$ ).

Cette absence de reconnaissance sociale de la communauté chinoise par les pouvoirs publics français accentue la marginalisation des individus qui ne peuvent pas avoir un 
accès direct à la société française. Paradoxalement cela tend à renforcer la dépendance des plus démunis vis-à-vis de cette communauté.

\section{Quand la communauté rend vulnérable}

Au-delà de la non-reconnaissance de la communauté par la société d'accueil, le repli sur les semblables a un coût: la dépendance à la communauté. Cette vie exclusivement intra-communautaire renforce la vulnérabilité de la population. Tout d'abord, la vie quotidienne se limite aux offres proposées par la communauté. Dans ce contexte de manque d'alternative, certains patrons, logeurs ou interprètes, profitent et exploitent la pénurie de ressource de leurs compatriotes ${ }^{12}$.

Le marché de l'emploi ethnique se caractérise par une grande précarité. Les opportunités sont limitées et les conditions de travail et de salaires sont inférieures à ce qui est pratiqué en France tant au niveau de l'emploi légal qu'informel. Il semble qu'il y ait une entente tacite entre employeurs et employés pour laisser de côté les normes et protections françaises et se référer plutôt à des pratiques inspirées de la Chine. Les salaires sont extrêmement bas et sans lien avec le nombre d'heures travaillées. Dans la confection, il n'est pas rare de voir des employés, payés à la pièce, travaillant 12 à 15 heures par jour dans des ateliers clandestins installés dans des locaux non aérés ou des caves, pour un salaire moyen de $800 €$ par mois. Les nourrices à domicile dénoncent des temps de travail particulièrement long et même extensibles car elles peuvent être sollicitées de 7 heures du matin à plus de minuit, sept jours sur sept, contre une rémunération mensuelle de 600 à 900 euros.

Nous avons pu constater lors de nos terrains que le logement subit également ce déclassement communautaire. Les conditions d'hébergement sont sommaires et pour le plus grand nombre en dortoirs surpeuplés et aménagés de manière rudimentaire. Il n'est pas rare de trouver 8 personnes dans une chambre de $12 \mathrm{~m}^{2}$, chaque couchette de lit superposé étant facturée autour de 110 euros par mois. On construit des lits superposés doubles pour les couples. Les chambres sont souvent mixtes et l'intimité n'est préservée que par de minces rideaux. Les gens font la queue pour avoir accès aux toilettes et à la cuisine et certains logeurs veillent à une consommation très réduite d'eau et d'électricité. Beaucoup de migrants se plaignent des appartements insalubres, humides et non chauffés en hiver et les mettent en relation avec la dégradation de leur état de santé.

L'accès à l'information est extrêmement limité. Elle provient généralement des pairs et reste peu fournie et parcellaire. C'est un terrain idéal pour la propagation de rumeurs, comme par exemple autour des jours de rafles policières ou des conditions de régularisation. De fait, l'information en provenance de la société française est souvent invérifiable et passe nécessairement par le biais d'intermédiaires : ceux qui parlent le français, qui jouissent d'un statut privilégié mais ne sont pas toujours fiables et/ou honnêtes. Dans le meilleur des cas, les informations sont de première main et proviennent de membres de la famille ou d'amis parlant le français, cependant aucun élément ne garantit leur réel niveau de compréhension de l'information ni du mode de fonctionnement de la société et des administrations françaises. Ces difficultés d'accès à l'information ne facilitent pas les échanges et la compréhension du pays d'accueil et de son mode de fonctionnement. La majorité des migrants ignore les us et coutumes, les institutions et les lois françaises. Ils ont tendance lors des interactions avec la société d'accueil à reproduire des manières de faire proprement chinoises. Or, si celles-ci ont 
fait leurs preuves en Chine, elles sont en revanche peu efficaces en France et attirent parfois le ressentiment des Français. C'est par exemple le cas lorsque les migrants qui ne sont pas habitués à la gratuité de l'aide humanitaire ou sociale, proposent de l'argent aux bénévoles d'associations ou aux assistantes sociales.

Enfin, contrairement à l'impression qu'en a la société d'accueil, la communauté chinoise n'est pas une entité homogène unie fortement par des relations de solidarité et d'entraide. Au contraire, des clivages s'installent en son sein selon les origines régionales des individus. Plusieurs facteurs expliquent cette diminution des capacités intégratrices de la communauté face à l'augmentation importante du nombre de migrants et de la diversification de leur provenance. La conjugaison de la crise économique et de la lutte contre l'immigration clandestine et le travail informel ont conduit à une raréfaction des emplois et à une précarisation généralisée des situations. Dans ce contexte, la concurrence en interne a mis à mal les différentes solidarités. On constate l'existence d'une fracture intra-communautaire sur des bases géo-dialectales. En effet, les personnes en provenance du Nord, arrivées récemment, ne sont pas insérées dans les réseaux d'entraide tissés depuis des décennies par les Wenzhou, beaucoup plus nombreux et mieux établis en France. L'exclusion se structure autour du dialecte, premier élément d'identification des personnes. Les Wenzhou, dont la langue est incompréhensible aux autres Chinois, l'utilisent comme instrument de distinction et d'exclusion. Cette ségrégation se combine à une domination hiérarchique : les Wenzhou sont en position d'employer les gens du Nord. Les inimitiés sont très vives, "acides »: "ils ne nous considèrent pas comme des êtres humains». Cependant, même au sein de groupes géo-dialectaux structurés, comme pour les Wenzhou, les témoignages laissent penser que les cercles d'entraide se resserrent sur la famille ou les très proches. Les récits d'exploitation entre membres d'une même famille ne sont pas rares, tel celui d'une tante ayant insisté pour faire venir sa nièce en France, la faisant ensuite travailler dans son atelier dans des conditions proches de l'esclavage pour rembourser la dette puis, lorsque celle-ci s'échappe, la remplaçant par une sœur. Ces phénomènes sont encore l'objet d'une relative tolérance de la part des Wenzhou qui s'accordent tout en les déplorant, à expliquer ces situations par « la dureté de la vie en France».

On le voit, le sas communautaire qui représente au moins dans les premiers temps de l'installation en France une ressource exceptionnelle, peut devenir à moyen terme un milieu enfermant et précarisant. Certains migrants cherchent alors à se tourner vers la société française.

\section{Les réponses à la vulnérabilité}

32 De part et d'autre les acteurs font le constat du problème de communication et s'organisent. En dehors de l'apprentissage de la langue par les migrants eux-mêmes, engouement dont nous avons vu les limites, plusieurs types de réponse existent.

\section{Les réponses de la société française}

Du côté français, la surprise de découvrir un public non francophone et la gêne peuvent même faire place à l'exaspération. Nous avons pu être témoins, lors d'accompagnements et de traductions, de l'incompréhension de certains acteurs sociaux (assistantes sociales, instituteurs ou médecins) qui s'interrogent régulièrement 
sur l'opportunité pour les migrants de rester vivre en France étant donné que leur incapacité à communiquer s'ajoute à leurs difficultés économiques et sociales. Nord, se présentent dans les administrations françaises sans traducteurs ou s'en remettent aux services payants de traducteurs ou de cabinets juridiques chinois. Ces derniers n'hésitent pas à tirer profit de la dépendance et de la crédulité des gens en facturant abusivement toute sorte de démarches. En effet, de nombreux moments de la vie quotidienne sont conditionnés par le degré de maîtrise du français, que cela soit avec des acteurs privés comme par exemple lors de la recherche d'un travail ou d'un logement, ou avec des services publics ou commerciaux, tels que l'école, la poste, les banques, les hôpitaux, les services sociaux. Voici des exemples de tarifs pratiqués : 10 euros pour la lecture-traduction d'une lettre, 30 pour la rédaction d'une réponse, entre 30 et 100 euros pour un accompagnement, 200 euros pour l'ouverture d'un compte en banque, une centaine d'euros pour la recommandation pour un emploi, un mois de «loyer " pour la présentation d'un logement ou d'un lit, 200 euros pour un certificat d'hébergement ou d'une adresse (nécessaire aux démarches auprès de l'OFPRA ${ }^{13}$ ) et de 1 000 à 3000 euros pour la présentation d'un homme français avec mariage à la clé. Obtenir une régularisation du statut de séjour est le service le plus cher.

\section{L'investissement dans les enfants}

Étant donné les difficultés d'apprentissage de la langue par les adultes, les parents misent sur les facilités linguistiques de leurs enfants, comme on a pu le voir dans les 
familles migrantes d'Afrique du Nord dans les années 1960 par exemple. En France, tous les enfants de moins de 16 ans, quelle que soit leur situation administrative de séjour ou celle de leurs parents, sont scolarisés gratuitement. l'Éducation Nationale prévoit un accueil spécifique des enfants migrants non-francophones de plus de six ans : ils sont scolarisés l'année de leur arrivée dans des classes pour non-francophones où ils apprennent le français de façon intensive, puis sont progressivement insérés dans des classes et cursus normaux.

La conception française de l'école comme moyen d'acquérir des connaissances générales nécessaires pour choisir un métier et élargir les opportunités des individus est parfois peu comprise par certaines familles chinoises Wenzhou ${ }^{14}$. Certains parents considèrent les enfants comme des acteurs du projet familial et ont parfois tendance à ne voir en l'école qu'un des moyens pour atteindre, par le biais de l'enfant, l'objectif d'enrichissement à moyen terme. Ainsi, l'enfant devient celui qui dans la famille apprend le français et peut servir d'intermédiaire avec la société d'accueil. Dès lors, il arrive que certains élèves soient déscolarisés dès qu'ils ont acquis une connaissance correcte de la langue, pour aider et travailler au commerce des parents. En deçà de ces cas extrêmes, beaucoup de professeurs signalent le manque de disponibilité pour les études des enfants. En effet, les jeunes adolescents primo-arrivant sont fréquemment sollicités; certains travaillent en rentrant de l'école pour rembourser leur dette de passage, d'autres servent de traducteurs ou d'accompagnateurs lors des démarches administratives. Ils jouent ainsi un rôle crucial pour la famille et la communauté lors de demande de papiers à la préfecture, d'ouverture de compte en banque ou d'accompagnement à l'hôpital, et sont, malgré eux, investis d'un statut et de responsabilités parfois disproportionnés par rapport à leur âge ${ }^{15}$.

Par ailleurs, le regard porté par les parents sur la scolarisation des enfants a des implications évidentes sur la qualité et la durée de l'apprentissage. Lorsque les parents s'investissent peu dans la scolarité des enfants, les résultats scolaires s'en ressentent, comme le signalent les professeurs qui constatent de grandes difficultés d'apprentissage, voire des échecs. Malgré leurs facilités dues à leur âge et à la nonrupture de leur parcours de scolarisation, certains adolescents n'arrivent pas à maitriser parfaitement la langue, ce qui ne leur permet pas d'envisager des projets d'avenir extérieurs à la socialisation proposée par leur communauté. Ils se destinent alors aux secteurs des trois couteaux (confection, restauration, maroquinerie) propres à l'économie ethnique, reprenant par là les projets migratoires et la vision de réussite de leurs parents ${ }^{16}$.

\section{Conclusion : l'obstacle linguistique, révélateur d'un hiatus entre différents projets migratoires ?}

40 Les difficultés rencontrées par les Chinois pour apprendre le français deviennent en France un facteur majeur de vulnérabilité. «En France, je suis devenue une aveugle sourde et muette; nous sommes des handicapés. » Ne pouvant accéder de façon individuelle à la société d'accueil, ils sont contraints de vivre dans un sas communautaire qui devient, pour certains, enfermant. Toutefois, l'obstacle linguistique met également en lumière une autre réalité de cette immigration dans la société française : il est le révélateur d'un hiatus entre le projet migratoire des migrants et le projet politique de la nation française. 
41 On peut se demander, en effet, comment les migrants chinois perçoivent le projet d'intégration de la République française. Cette préoccupation semble leur être tout d'abord étrangère. D'après leurs discours, leur objectif dans la migration est clair et avant tout économique, en particulier pour les Wenzhou. La France n'est qu'un lieu parmi d'autres pays d'implantation de la diaspora chinoise où ce projet d'enrichissement est possible. Il ressort des entretiens qu'il ne s'agit moins d'un choix effectué en fonction de supposées "valeurs françaises" mais plutôt au regard de situations sociales spécifiquement chinoises. À l'instar d'autres migrants économiques ou réfugiés, les Chinois, qu'ils soient originaires du Nord ou du Sud de la Chine, n'ont pas eu vraiment le choix du pays de destination. Il s'agit d'un choix contraint qui est, pour les migrants du Nord sans attaches en France, pris plutôt par les passeurs ou selon des considérations budgétaires ${ }^{17}$. Les Wenzhou, quant à eux, se dirigent vers la France quand ils ont de la famille ou des amis qui peuvent les accueillir et les aider sur place.

42 Les témoignages laissent également penser que les migrants semblent moins préparés à l'aspect immigration, qu'à celui d'émigration. Le vocabulaire employé apporte, à cet égard, un éclairage sur la manière dont ils envisagent leur expérience migratoire. Le terme de "migration » yimin, est très peu utilisé, alors que les personnes se réfèrent à leur parcours sous le terme de chuguo "sortir du pays». Ce mot met l'emphase sur l'aspect de départ et de vie en expatriation loin du Pays plutôt que sur le parcours d'installation dans un autre pays. Il semble en effet que peu de migrants aient réellement réfléchi avant leur départ au fait qu'ils allaient vivre dans un pays où ils seraient alors en position d'étranger. La surprise de certaines personnes découvrant à leur arrivée qu'elles ne peuvent pas se faire comprendre par les Français faute de connaissance linguistique, met en lumière leur manque de préparation. Il semble que l'objectif économique ait primé sur les autres aspects de l'expérience de l'expatriation et rares sont ceux qui avaient envisagé leur projet migratoire comme une expérience de l'altérité, de découverte d'une autre culture. Une fois en France, les migrants sont soumis à des contraintes telles qu'ils n'ont plus la disponibilité suffisante pour faire évoluer leur compréhension de la société d'accueil. D'ailleurs, dans les discours, les Français sont désignés sous le terme d'étranger, waiguoren. Tout laisse à penser qu'ils sont donc loin d'envisager leur séjour sous l'angle de l'intégration, au sens où l'entend la société française.

Bien des éléments développés ici ne sont pas spécifiques de cette migration. Ils mettent en lumière le hiatus entre des individus qui viennent en France pour gagner de l'argent sans se préoccuper plus avant de la problématique d'intégration chère à la société d'accueil, et une société qui attend des immigrés une adhésion idéologique à ses valeurs et à son projet politique. Les difficultés d'apprentissage linguistique chez les Chinois d'un côté, et le discours volontariste d'intégration produit par la République française de l'autre, dévoilent le grand écart qui conduit à une incompréhension mutuelle et à une marginalisation grandissante de ces primo-arrivants en France. 


\section{BIBLIOGRAPHIE}

AUGUIN Estelle (2005) « Le lien filial en migration : les Chinois du sud du Zhejiang », in Hommes \& Migrations (1254), pp. 18-28.

CATTELAIN Chloé (Éds) (2002) Les modalités d'entrée des ressortissants chinois en France, Paris, Direction de la Population et des Migrations, Ministère de l'Emploi, de la Solidarité et de la Cohésion sociale (non publié).

COSTA-LASCOUX Jacqueline (2006) «L'intégration « à la française » : une philosophie à l'épreuve des réalités », in Revue Européenne des Migrations Internationales, 22 (2), pp. 105-126.

GAO Yun, POISSON Véronique (2005) Le trafic et l'exploitation des immigrants chinois en France, Genève, Bureau International du Travail.

GAO Yun, LEVY Florence, POISSON Véronique (2006) « De la migration au travail, l'exploitation extrême des chinois-e-s à Paris », in Travail, genre et sociétés (16), pp. 53-74.

MORENO Sabine (2005) « Le projet « Chinois d'Europe et intégration » », Hommes \& Migrations (1254), pp. 58-73.

PINA-GUERASSIMOFF Carine, GUERASSIMOFF Eric, WANG Nora (2002) La circulation des nouveaux migrants économiques chinois en France et en Europe, Paris, Ministère de l'Emploi, de la Solidarité et de la Cohésion sociale, DRESS/MIRE, (non publié).

SAILLARD Claire, BOUTET Josiane (2001) Pratiques des langues chez les jeunes issus de l'immigration chinoise à Paris, Délégation Générale à la Langue Française (non publié).

SCHNAPPER Dominique (1991) La France de l'intégration. Sociologie de la nation en 1990, Paris, Gallimard, 367 p.

SCHNAPPER Dominique (2000) Qu'est-ce que la citoyenneté ?, Paris, Gallimard, 320 p.

THIESSE Anne-Marie (1999) La création des identités nationales. Europe XVIII ${ }^{\mathrm{e}}-\mathrm{XX}{ }^{\mathrm{e}}$ siècle, Paris, Seuil, $305 \mathrm{p}$.

WEBER Eugen (1983) La fin des terroirs. La modernisation de la France rurale 1870-1914, Paris, Fayard, 844 p., [première édition originale : 1976].

\section{NOTES}

1. Il est important de garder à l'esprit toute l'ambiguïté de cette notion de "communauté ». Celle-ci est perçue par la société française comme un groupe relativement homogène et lié par des liens de solidarité et d'entraide forts. Or, les témoignages montrent que la plupart des migrants chinois ne perçoit pas les choses de cette manière et n'utilise pas ce terme, qui dès lors semble être une définition plaquée de l'extérieur. Cependant, nous emploierons ce terme par commodité pour décrire ce groupe social composé des ressortissants chinois installés en France tout en sachant qu'il est loin d'être homogène mais est au contraire traversé par de forts clivages, en particulier perceptibles par la langue.

2. Les auteures mènent deux enquêtes de terrain dans le cadre de leur recherche de thèse commencée depuis 2003. Outre des entretiens semi-directifs, un long travail d'observation participante est entrepris à travers des activités associatives auprès d'un public d'adolescents et 
d'adultes chinois, telles que: soutien scolaire, accompagnement social, interprétariat, enseignement du français, travail social de rue, etc.

3. Nous excluons ici les boat-people réfugiés d'Asie du Sud-est à la fin des années 70 et souvent qualifiés hâtivement, en France, de "Chinois ». Ne bénéficiant pas des mêmes conditions de départ et d'arrivée que les migrants directement originaires de République Populaire de Chine, ils n'ont pas rencontré les mêmes difficultés d'intégration et il est difficile de comparer leurs parcours. Nous n'étudierons pas non plus le cas des migrants originaires de RPC, venus de manière légale (étudiants, personnes très qualifiées, réfugiés politiques etc.). On constate que ces distinctions sont reprises par les acteurs et que ces différentes populations se mélangent peu à l'exception du cadre professionnel.

4. $88 \%$ des 300000 migrants légaux d'origine chinoise vivant en France habiteraient Paris et la petite couronne (Cattelain, 2002) et tout nous laisse penser que les sans-papiers se concentrent aussi essentiellement dans la région parisienne. Cependant, nous ne disposons par définition que de peu d'informations quantitatives fiables à leur sujet.

5. Étant donné la quasi impossibilité pour les personnes originaires de la région de Wenzhou d'obtenir un visa d'entrée pour la France, elles se tournent principalement vers des passeurs et entrent puis séjournent de façon illégale sur le territoire français. Ils espèrent, à l'instar de leurs prédécesseurs, obtenir au bout de quelques années de séjour en France une régularisation de leur statut. Les Dongbei entrent sur le territoire avec des visas commerciaux de courte durée et restent après l'expiration de leur visa.

6. Cette première initiative des pouvoirs publics de définition du projet politique du pays s'inscrit aussi dans un contexte idéologique de contrôle des migrants.

7. Rappelons que leur langue maternelle est un dialecte régional plus ou moins proche du mandarin, seule langue officielle reconnue depuis 1949, et qu'ils ne l'apprennent souvent qu'à l'école. Voir Saillard et Boutet, 2001.

8. Claire Saillard et Josiane Boutet rappellent que « une des particularités du chinois en fait un moyen de communication unique : l'écriture chinoise n'encode pas des sons, mais des concepts. » $(2001: 12)$

9. Voir l'article «Adresse : septième arrêt du bus 393, deuxième rue à droite ", dans Courrier International numéro spécial: Destins d'émigrés, $\mathrm{n}^{\circ} 8$ 14, juin 2006: 41. Bien entendu ces stratégies ne sont pas spécifiques de ce groupe mais également mises en œuvre par d'autres populations, notamment les personnes non alphabétisées.

10. Les enfants sont scolarisés dans une école française. Toutefois, durant la première année de scolarisation, beaucoup suivent des cours de français supplémentaires dans des écoles communautaires le soir après l'école, les mercredi et samedi après-midi ainsi que durant les vacances scolaires. De fait, l'emploi du temps en Chine est chargé et beaucoup de Chinois considèrent que les enfants ne travaillent pas assez à l'école française.

11. Ce projet évolue rapidement, en particulier pour les femmes divorcées, qui souhaitent finalement s'établir en France et voient dans leur séjour l'occasion de refaire leur vie et de se (re) marier avec des hommes français.

12. Sur les conditions d'exploitation extrême voir Gao et Poisson, 2005.

13. Office Français de Protection des Réfugiés et Apatrides.

14. Pour plus de détail sur ce sujet voir Auguin, 2005. Ces attitudes que l'on a pu observer dans d'autres migrations ouvrières, se distinguent de celles des familles migrantes d'Asie du Sud-est qui ont au contraire misé majoritairement sur l'insertion par l'école française. La dimension sociale apporte un éclairage sur ces différences.

15. De nombreuses études sur les enfants de migrants montrent que ce phénomène, comme celui de la participation des enfants au projet parental n'est en rien proprement chinois. Ceci est par exemple repris dans la communication non encore publiée de Christine Catarino et Laura Oso «Labour culture and Labour Commitment of Children of Immigrant Entrepreneurs in France and 
Spain » lors de la conférence Gender, Generation and the Family in International Migration. Florence, 14-16 juin 2007.

16. Sortir du marché ethnique du travail n'est pas forcément considéré par les parents Wenzhou comme un critère de réussite. Ce point de vue des intéressés contraste fortement avec celui des pays d'accueil, qui ont par exemple lancé des projets visant notamment à diversifier les débouchés des populations chinoises en France, Allemagne et Italie (projet européen EQUAL). Voir à ce sujet S. Moreno, 2005.

17. Les entretiens montrent que la France est une destination de deuxième choix. Le prix du passage est un tiers moins élevé que celui vers les destinations plus prisées telles que les ÉtatsUnis ou le Canada.

\section{RÉSUMÉS}

À partir de l'arrivée massive des migrants d'origine asiatique et chinoise au milieu des années soixante-dix jusqu'à une période récente, l'immigration chinoise pose peu de problème à la société française qui l'observe comme discrète et économiquement bien intégrée. Cependant, depuis la fin des années quatre-vingt-dix, la société voit apparaitre des signes de difficultés. Les ressources internes à la communauté ne semblent plus suffisantes pour résoudre l'ensemble des problèmes de ses membres et, de plus en plus de personnes se tournent vers la société française qui découvre une population vulnérable. L'hypothèse principale de cet article est de considérer, qu'étant donné l'importance attribuée à la maitrise de la langue française dans le modèle d'intégration français, le facteur linguistique est central pour comprendre la vulnérabilité des Chinois en France. En effet, la maîtrise de la langue française conditionne la sociabilité des migrants dans la société d'accueil et leur accès direct à cette dernière. En analysant le projet politique de la République française en matière d'immigration et le projet migratoire des Chinois en France, l'article, fondé sur deux études de terrain, dévoile un hiatus entre les deux projets, qui fragilise cette population migrante.

Language and Vulnerability among Current Chinese Migrations. From the massive arrivals of migrant of Asian and Chinese origins in the mid 1970's, Chinese immigration did not create until recently problems to the French society which regarded it as discrete and economically wellintegrated However from the end of the 1990's, some problems appear. The inner resources of the community do not seem sufficient to solve the problems encountered by their members and more and more migrants turn to the host society, which discovers that they constitute a vulnerable population. The main hypothesis of this paper states that because of the importance granted by the French model of integration to mastering French, language is a major factor of vulnerability, which commands their access to French society and the whole of their sociability process. The article, which is based on two field studies, shows that the gap between the French republican model of immigration and the projects of the Chinese immigrants is a source of fragility.

Lengua y vulnerabilidad de las migraciones chinas actuales. Desde mediados de los años setenta llegan masivamente inmigrantes de origen asiático y chino a Francia, inmigración que ha generado pocos problemas a la sociedad francesa que la considera discreta y económicamente bien integrada. Sin embargo, desde finales de los años noventa, la sociedad asiste a la aparición 
de signos de dificultad. Los recursos internos de la comunidad ya no parecen suficientes para resolver el conjunto de problemas de sus miembros y, cada vez más personas, se vuelven hacia la sociedad francesa la cual descubre una población vulnerable. La hipótesis principal de este artículo es considerar que, dada la importancia atribuida al dominio de la lengua francesa en el modelo francés de integración, el factor lingüístico es central para comprender la vulnerabilidad de los chinos en Francia. En efecto, el dominio del francés condiciona la sociabilidad de los inmigrantes en la sociedad de acogida y su acceso directo a ésta. Analizando el proyecto político de la República francesa en materia de inmigración y el proyecto migratorio de los chinos en Francia, el artículo, fundado en dos estudios de terreno, desvela un hiatos entre los dos proyectos que fragiliza a esta población inmigrante.

\section{AUTEURS}

\section{ESTELLE AUGUIN}

Doctorante, UMR CEPED (Paris Descartes-INED-IRD), 45 rue des Saints Pères 75006 Paris. estelle.auguin@paris5.sorbonne.fr

\section{FLORENCE LEVY}

Florence Lévy, doctorante en sociologie, Centre d'études sur la Chine moderne et contemporaine, EHESS - MAPS, Université de Neuchâtel, florence.levy@unine.ch 